\title{
Habilidades Sociais de Estudantes de Psicologia: Um Estudo Multicêntrico
}

\author{
Zilda A P. Del Prette ${ }^{12}$ \\ Almir Del Prette \\ Maria Cecília Mendes Barreto \\ Universidade Federal de São Carlos \\ Marina Bandeira \\ Universidade Federal de São João Del Rei \\ Maria Refugio Rios-Saldaña \\ Universidade Nacional Autônoma do México \\ Ana Lucia Alcântara Oliveira Ulian \\ Universidade Federal da Babia \\ Eliane Gerk-Carneiro \\ Universidade Gama Filho \\ Eliane Mary de O. Falcone \\ Universidade Estadual do Rio de Janeiro \\ Miriam Bratisch Villa \\ Universidade de São Paulo, Ribeirão Preto
}

\begin{abstract}
Resumo
Habilidades sociais que ocorrem com alta freqüência e pouca variabilidade podem ser consideradas como padrões, sugerindo características da cultura ou subcultura que são importantes na seleção de instrumentos de avaliação e no planejamento de intervenções. Este trabalho buscou caracterizar o repertório de habilidades sociais de estudantes de Psicologia com base em amostras de 4 localidades: São Paulo (SP), Bahia (BA), Minas Gerais (MG) e Rio de Janeiro (RJ), verificando diferenças entre elas, inclusive em termos da influência do sexo e idade. Quinhentos e sessenta e quatro estudantes responderam 1 inventário de auto-relato IHS-Del-Prette \& A. Del Prette, 2001, cujos itens agrupavam-se em 5 fatores: F1) enfrentamento e auto-afirmação; F2) expressão de afeto positivo; F3) conversação e desenvoltura social; F4) auto-exposição a desconhecidos e situações novas; e F5) autocontrole da agressividade. Com base no escore geral (EG) e escores fatoriais (F1, F2, Fe, F4 e F5), foram efetuadas análises comparativas por localidade e, em cada uma delas, por sexo e idade (ANOVA seguida de teste tou Scheffé). Os resultados indicaram que: a) os estudantes de Psicologia apresentaram escores mais altos que os da amostra normativa no EG, F1, F3 e F4 e inferiores no F2 e F5, mas as diferenças de sexo seguiram os padrões normativos; b) houve influência da localidade, sexo e idade sobre os escores, com interação local-sexo e local-idade; c) as amostra de MG, BA, RJ e SP apresentaram perfil semelhante para F3 e F4, mas RJ apresentou escores mais altos no F1, MG e SP no F2 e F5; d) houve mais diferenças de gênero na BA e menos no RJ; e) as diferenças de idade ocorreram no F4 (favoráveis aos mais novos para SP) e no F5 (favoráveis aos mais velhos para BA e RJ). Discute-se as diferenças e semelhanças encontradas, levantando-se hipóteses e questões para pesquisa adicionais.
\end{abstract}

Palavras-chave: Habilidades sociais; cultura; estudantes de Psicologia; escalas de avaliação.

\section{Social Skills of Psychology Undergraduates: A Multicentered Study}

\begin{abstract}
Social skills occurring in a high frequency and low variability can be taken as patterns, suggesting cultural or sub-cultural features which are important when selecting instruments for evaluation and planning interventions. This research aimed to characterize the social skills repertoire among Psychology students from 4 Brazilian places: São Paulo (SP), Bahia (BA), Minas Gerais (MG)e Rio de Janeiro (RJ), also examining their differences and the influence of sex and age on their patterns. Five hundred sixty four Psychology undergraduates completed a self-report inventory (IHS-del-Prette) with a structure of 5 factors: F1) coping and assertion; F2) expressing positive affection; F3) talking and selfconfidence; F4) dealing with unknown people and new situations; F5) aggressiveness self-control. The samples were compared considering general (GS) and factorial (F1, F2, F3, F4 e F5) scores as well as sex and age influence in each one (ANOVA, followed by t or Scheffé test). The results showed that: a) Psychology students presented higher scores than normative sample in the GS, F1, F3 and F4 and lower inthe F2 and F5, but sex differences were similar to the normative pattern; b) place, sex and age significantly affected the scores, with place-sex and place-age interactions; c) samples from MG, BA, RJ and SP presented similar profiles, with higher values for RJ in the F1 and for MG and SP in the F2 and F5; d) students from BA presented the highest sex differences and from $\mathrm{RJ}$ the lowest ones; $\mathrm{e}$ ) age differences occurred in the $\mathrm{F} 4$ (favorable to younger students for SP) and in the F5 (favorable to older ones for BA and RJ). The meaning of these differences and similarities are discussed as well as some explanatory hypotheses and questions for further studies.

Keywords: Social skills; culture; Psychology undergraduates; evaluation scales.
\end{abstract}

\footnotetext{
${ }^{1}$ Trabalho originalmente apresentado no Simpósio: "Habilidades sociais em estudantes universitários: Um estudo transcultural", na XXXI Reunião Anual da Sociedade Brasileira de Psicologia, em 2001. Os autores agradecem aos professores e alunos de Psicologia, das várias localidades, que colaboraram com na coleta dos dados deste trabalho. E também a um dos pareceristas que, mesmo anônimo, apresentou sugestões valiosas que contribuíram no aperfeiçoamento final do texto.

${ }^{2}$ Endereço para correspondência: Alameda das Ameixeiras, 60, Parque Faber, 13570 970, São Carlos, SP.
} 
A avaliação do repertório de habilidades sociais pode focalizar aspectos observáveis ou não observáveis do comportamento. Os aspectos observáveis referem-se às classes comportamentais amplas ou molares como fazer e responder (a) cumprimentos e elogios, expressar opiniões e discordâncias, iniciar, manter e encerrar conversações, fazer críticas e responder a elas etc. e, também, a seus componentes moleculares verbais e não-verbais, como de tom de voz, contato visual, gestos, postura etc. Os aspectos não diretamente observáveis incluem pensamentos, percepções, representações etc., que precedem, acompanham ou seguem o desempenho social.

Dada a amplitude das dimensões associadas aos conceitos de habilidades sociais e de competência social, justifica-se a existência de vários métodos de avaliação. Os instrumentos usuais de avaliação incluem o relato por significantes (inventários, questionários, roteiros de entrevistas), a observação direta (registros cursivos de episódios ou classes específicas de comportamentos, em situação natural e/ou em situações estruturadas de desempenho de papéis) e o auto-relato (inventários, questionários e roteiros de entrevistas).

Os registros de observação fornecem importantes subsídios para se examinar a funcionalidade do desempenho social em diferentes contextos e com diferentes interlocutores, mas requerem uma sofisticada metodologia de análise (um exemplo dessa metodologia pode ser encontrado em Bandeira, 1999; Bandeira \& Tremblay, 1998), que restringe sua aplicação em larga escala com objetivos normativos. Nesse caso, o instrumento mais viável, e mais comumente utilizado, tem sido o inventário, com questões que contemplam uma diversidade de demandas, interlocutores e situações.

Embora o máximo da competência social implique em uma maior flexibilidade comportamental, própria da capacidade adaptativa do ser humano, as pessoas não são igualmente "habilidosas" nas diferentes situações interpessoais e os desempenhos socialmente aprovados e valorizados podem variar bastante de uma cultura para outra. Esse caráter situacionalcultural da competência social opõe-se à noção de "traços de personalidade" (McFall, 1982), implicando na necessidade de se avaliar o desempenho social sob diferentes contextos, demandas e interlocutores prováveis e significativos nas relações interpessoais da população alvo.

Em relação ao caráter cultural, a literatura destaca a influência dos valores, normas e expectativas sobre os desempenhos sociais da população em geral e de subgrupos com características sóciodemográficas específicas em termos de sexo, idade, ocupação e papéis sociais etc. (Argyle, 1984, 1994; Bresnahan, Sherman \& Lee, 2002; Caballo, 1993; A. Del Prette. \& Z. Del Prette, 1999, 2001; Fray \& Hector, 1987; Furnham, 1979, 1993; Trower, Bryant \& Argyle, 1978), que podem constituir subculturas com seus padrões próprios de condutas aceitáveis. Dentre esses aspectos, sexo e idade têm sido os mais críticos, levando a estudos sobre padrões desenvolvimentais e de gênero que devem necessariamente ser diferenciados quando se buscam normas de referência. As diferenças na competência social de homens e mulheres ocorrem desde a infância (LaFrenière \& cols., 2002), embora localizadas em fatores ou itens específicos. Caballo (1993) cita vários estudos que mostram as mulheres como mais habilidosas na expressão de sentimentos positivos e os homens na expressão de sentimentos negativos e na habilidade de fazer solicitações, o que também se verificou em estudos realizados no Brasil (Z. Del Prette \& A. Del Prette, 2001). Em resumo, as variáveis sócio-demográficas afetam o desempenho social, porém o peso dessa influência pode ser diferente sob contextos culturais diversos.

A freqüência com que determinadas habilidades são emitidas pelos indivíduos de um grupo ou contexto social constitui um indicador dos comportamentos efetivos e valorizados nesse contexto e pode ser tomada como referência para avaliar o ajuste ou afastamento, de um indivíduo específico, das normas e expectativas de seu grupo. $\mathrm{O}$ afastamento pode ser resultado tanto de déficits de observação, percepção social, discriminação e controle sobre o próprio comportamento como de atitudes, concepções e valores divergentes do padrão predominante na cultura do próprio grupo. Certos indivíduos, ao se mostrarem inovadores e, mesmo, idiossincráticos, podem produzir mudanças nos padrões sociais e, também, nos valores e atitudes de seus grupos. Um exemplo foi o movimento hippie dos anos 1960, considerados como contracultura na época e posteriormente incorporados pela sociedade.

A busca dos padrões normativos de uma dada cultura e de variantes das subculturas nela existentes tem levado a estudos transculturais usualmente direcionados para a adaptação e validação de instrumentos de avaliação do repertório de habilidades sociais ou com objetivos de orientar intervenções visando a convivência e o ajustamento de indivíduos em diferentes culturas (Ex.: Bresnaham, Shearman \& Lee, 2002; Carmona \& Lorr, 1992; Fray \& Hector, 1987; Furnham, 1979, 1993; Nikura, 1999; Wood \& Mallinckrodt, 1990; Yashima \& Tanaka, 2001). Além desses objetivos, defende-se que a análise comparativa dos padrões de habilidades sociais em diferentes culturas ou subculturas pode ser explorada para, em sentido oposto, subsidiar inferências válidas sobre as normas e valores aí predominantes e como um caminho para se identificar padrões mais generalizados de desempenhos considerados socialmente

\footnotetext{
${ }^{3}$ O leitor poderá encontrar em Hall (1977) uma descrição da variabilidade de comportamentos interpessoais em diferentes culturas, principalmente comparando as culturas ocidental e oriental. Esforços mais recentes em direção semelhante porem com sob um outro referencial teórico podem ser localizados na chamada Psicologia Transcultural (Ex.: ver Lonner, Dinnel, Hayes \& Sattler, 2002).
} 
competentes, não obstante a especificidade situacional sob cada uma delas 3

A diversidade cultural, amplamente reconhecida em nosso país e representada no cinema, na literatura, na música e em estudos antropológicos e sociológicos, sugere costumes, valores e crenças que podem afetar as características do desempenho social de subculturas regionais. Embora fundadas em um certo folclore e reforçadas por estereótipos, a forma como se traduzem, no cotidiano das relações interpessoais, as diferenças de desempenho social entre nortistas, nordestinos, sulistas etc., ou entre mineiros, gaúchos, cariocas, paulistas, baianos etc., não têm recebido muita atenção na literatura psicológica.

Assim, além de prover informações técnicas sobre as qualidades psicométricas dos instrumentos utilizados, a análise dessas diferenças pode ser importante na compreensão dos padrões de relacionamento predominantes sob essas subculturas e na inferência de valores e normas comuns, ou específicos, que influem no desempenho social. $\mathrm{O}$ controle das variáveis sexo e idade permitira, nesse caso, examinar as relação entre essas variáveis pessoais e as características contextuais ou culturais mais amplas.

Nos estudos de larga escala, uma população bastante estudada é a de estudantes universitários: nos anos 1970 e 80 especialmente nos Estados Unidos (Chandler, Cook \& Dugovics, 1978;J.Galassi, Delo, M. Galassi \& Bastien, 1974) e na Inglaterra (Argyle, 1984, 1988; 1994; Bryant \& Trower, 1974); posteriormente, também em outros países (Abarca \& Hidalgo 1989; Caballo, 1995; Hidalgo \& Abarca, 1990), incluindo-se o Brasil (Ayres, 1994; A. Del Prette, 1978; Z. Del Prette \& A. Del Prette, 1983; Falcone, 1998). Esses estudos adquirem particular importância quando se considera que a formação de terceiro grau deveria incluir o desenvolvimento interpessoal como parte dos objetivos acadêmicos (Z. Del Prette \& A. Del Prette, 1983), principalmente naquelas áreas cuja atuação depende, criticamente da qualidade das relações profissional-cliente (A. Del Prette, A. \& Z. Del Prette, 2001; A. Del Prette, Z. Del Prette \& Branco, 1992a). Esse é o caso, por exemplo, da formação em Psicologia.

Em estudos nacionais sobre o desempenho interpessoal de universitários em nosso meio (Del Prette, Del Prette \& Branco, 1992a, 1992b; Z. Del Prette, A. Del Prette \& Correia, 1992) foi possível constatar alguns focos de dificuldades interpessoais generalizadas, bem como particularidades nos estudantes de Ciências Humanas, mais especificamente, de Psicologia. Os dados evidenciaram que os estudantes de Psicologia, ao entrarem para a Universidade, apresentavam um padrão indiferenciado dos demais, porém, ao longo do curso, pareciam desenvolver, de modo informal, uma maior "sensibilidade" para os relacionamentos interpessoais, sem indicadores de maior competência para lidar com essas situações.

Parte dos estudos realizados com estudantes universitários no Brasil utilizou o Inventário de Habilidades Sociais, ou IHS-
Del-Prette (Z. Del Prette \& A. Del Prette, 2001), produzindo evidências de propriedades psicométricas desse instrumento. Em termos de estabilidade temporal e correlação com outras medidas, um estudo de intervenção (A. Del Prette, Z. Del Prette \& Barreto, 1999), com medidas pré e pós-intervenção tomadas a um intervalo de 6 meses, os resultados do IHSDel-Prette indicaram diferença significativa para o grupo experimental, mas não para o grupo controle, o que foi coerente com dados de observação direta do desempenho dos participantes e seus relatos ao longo das sessões. A validade do IHS-Del-Prette foi verificada em outros estudos em termos de consistência interna e correlação com outras medidas de habilidades sociais (Gerk-Carneiro \& cols., 2001a, 2001b) e até de fatores de personalidade (Bueno, S. Oliveira \& J. Oliveira, 2001). Um estudo com a versão de 42 itens (Bandeira, Costa, Z. Del Prette, A. Del Prette \& GerkCarneiro, 2000) forneceu evidências adicionais de estabilidade teste-reteste $(r=0,90 ; p=0,001)$ e de validade concomitante $(r=0,79 ; p=0,01)$ com o Inventário de Assertividade de Rathus (1973). Embora não se disponha de dados sobre análise confirmatória da estrutura fatorial do IHS-Del-Prette, os estudos acima reportaram um satisfatório ajuste à estrutura fatorial encontrada na amostra normativa.

Dadas as considerações anteriores, o presente estudo teve por objetivos: a) caracterizar, com base no IHS-Del-Prette, o repertório de estudantes de psicologia de quatro localidades brasileiras (três da região sudeste e uma da região nordeste) e suas diferenças em relação à amostra normativa de universitários; b) analisar o efeito da localidade e das variáveis sócio-demográficas sexo e idade sobre cada uma dessas amostras; c) inferir características das subculturas locais, potencialmente relacionadas às diferenças eventualmente encontradas; d) examinar implicações dos resultados para as qualidade psicométricas do IHS-DelPrette e para novos estudos na área de Habilidades Sociais.

\section{Método}

\section{Participantes}

A amostra foi constituída por 564 estudantes de início e término do curso de Psicologia, de ambos os sexos (83\% mulheres e 17\% homens), idade média de 21,02 anos, oriundos de quatro localidades: 1) São Paulo (SP), com estudantes de São Carlos e Ribeirão Preto, a cerca de $300 \mathrm{~km}$ da capital; 2) Bahia (BA), da capital; 3) Minas Gerais (MG), de São João Del Rei, distante 190 $\mathrm{km}$ da capital; 4) Rio de Janeiro (RJ), da capital. Todos cursavam universidade pública, à exceção de uma parte da amostra do Rio de Janeiro.

A Tabela 1 apresenta as características sócio-demográficas da amostra em cada uma das localidades.

Como se observa na Tabela 1, há uma maior proporção de respondentes de SP (39\%), enquanto cada uma das outras 
Zilda A P. Del Prette, Almir Del Prette, Marina Bandeira, Maria Refugio Rios-Saldaña, Ana Lucia Alcântara Oliveira Ulian, Eliane, Gerk-Carneiro, Eliane Mary de O. Falcone, 344 Maria Cecília Mendes Barreto \& Miriam Bratfisch Villa

Tabela 1

Características Sócio-demográficas da Amostra Brasileira Avaliada

\begin{tabular}{|c|c|c|c|c|c|c|}
\hline \multirow{3}{*}{ Localidade } & \multirow{3}{*}{$N(\%)$} & \multirow{3}{*}{$\begin{array}{l}\text { Idade(anos) } \\
\text { Méd (DP) }\end{array}$} & \multicolumn{2}{|c|}{ Sexo } & \multicolumn{2}{|c|}{ Momento no Curso } \\
\hline & & & Masculino & Feminino & Início & Término \\
\hline & & & $\mathrm{N}(\%)$ & $\mathrm{N}(\%)$ & $\mathrm{N}(\%)$ & $\mathrm{N}(\%)$ \\
\hline $\mathrm{MG}$ & $100(0,18)$ & $21,60(2,37)$ & $14(0,14)$ & $86(0,86)$ & $50(0,50)$ & $50(0,50)$ \\
\hline $\mathrm{BA}$ & $132(0,23)$ & $21,12(1,89)$ & $31(0,23)$ & $101(0,77)$ & $58(0,44)$ & $74(0,56)$ \\
\hline SP & $219(0,39)$ & $20,42(1,73)$ & $34(0,15)$ & $184(0,85)$ & $125(0,57)$ & $94(0,43)$ \\
\hline RJ & $117(0,21)$ & $21,53(2,14)$ & $21(0,18)$ & $94(0,80)$ & $61(0,52)$ & $56(0,48)$ \\
\hline Total & $564(0,99)$ & $21,02(2,04)$ & $99(0,17)$ & $465(0,83)$ & $294(0,52)$ & $273(0,48)$ \\
\hline
\end{tabular}

Tabela 2

Matriz de Correlação entre as Variáveis Dependentes do Estudo

\begin{tabular}{cccccc}
\hline $\begin{array}{c}\text { Variável } \\
\text { Dependente }\end{array}$ & F1 & F2 & F3 & F4 & F5 \\
\hline Escore & $0,76^{* *}$ & $0,61^{* *}$ & $0,74^{* *}$ & $0,61^{* *}$ & $0,09^{*}$ \\
F1 & & $0,23^{* *}$ & $0,38^{* *}$ & $0,50^{* *}$ & $-0,01$ \\
F2 & & & $0,36^{* *}$ & $0,20^{* *}$ & 0,06 \\
F3 & & & & $0,37^{* *}$ & $0,09^{*}$ \\
F4 & & & & & 0,02 \\
\hline
\end{tabular}

Nota. ${ }^{* *}$ Correlação significativa a 0,01 (2-tailed); * Correlação significativa a 0,05 (2-tailed).

representa em torno de $20 \%$ da amostra total. A média geral de idade foi de 21 anos, com pequena variação: de 20,42 (SP) a 21,60 (MG). Em todas as amostras, apenas $1 / 4$ de respondentes é do sexo masculino, como é usual nos Cursos de Psicologia. A distribuição início-término é razoavelmente equilibrada em todas as amostras.

\section{Instrumento}

$\mathrm{Na}$ coleta de dados foi utilizado o Inventário de Habilidades Sociais, em uma versão de 42 itens que apresentou satisfatória consistência interna $(\alpha=0,8585 ; N=474)$. No entanto, como os estudos sobre a sua estrutura fatorial basearam-se na versão de 38 itens (quatro a menos), publicada como IHS-Del-Prette (Del Prette, Z. \& Del Prette, A., 2001), optou-se por analisar somente os dados referentes a esses 38 itens que, neste estudo, apresentaram consistênciainterna semelhante $(\alpha=0,8326 ; N=545)$.

O IHS-Del-Prette compõe-se de duas partes: a primeira contempla as instruções de preenchimento e a lista de itens, cada um deles descrevendo uma situação de demanda de desempenho social e uma possível reação a ela. Nas instruções, solicita-se que o respondente estime a freqüência com que reage da forma sugerida em cada item, considerando um total de 10 vezes em que se encontrou na situação descrita e indicando sua resposta com base em uma escala de cinco pontos (tipo Likert) que varia de nunca ou raramente (zero a $20 \%$ das vezes) a sempre ou quase sempre (81 a 100\% das vezes). Parte dos itens é redigida de modo que uma freqüência mais elevada indica déficit na habilidade requerida naquela situação (nesses itens a pontuação é invertida para a obtenção do escore). A segunda parte do IHS contém um cabeçalho para a coleta de informações sobre o respondente e uma tabela para a anotação das respostas, precedida pela escala de freqüência.

Os estudos com o IHS-Del-Prette indicaram uma estrutura de cinco fatores, com consistência interna satisfatória para a escala total (alfa de Cronbach $=0,75$ ) e para cada um desses fatores ou subescalas: F1) auto-afirmação e enfrentamento com risco $(\alpha=0,9650)$; F2) auto-afirmação na expressão de afeto positivo $(\alpha=0,8673) ; \mathrm{F} 3)$ conversação e desenvoltura social $(\alpha=0,8187)$; F4) auto-exposição a desconhecidos ou a situações novas $(\alpha=0,7525)$; F5) autocontrole da agressividade a situações aversivas $(\alpha=0,7413)$. A correlação entre o escore geral e os fatores bem como entre os quatro primeiros escores fatoriais do IHS-Del-Prette é altamente significativa $(p<0,01)$. Essas correlações foram também verificadas no presente estudo, conforme se apresenta na Tabela 2, sugerindo um ajuste satisfatório da estrutura fatorial prévia aos dados atuais.

\section{Procedimento de coleta de dados}

A coleta de dados foi realizada em grupo nas salas de aula. O aplicador expôs os objetivos da pesquisa, leu e explicou as instruções, enfatizou a ausência de respostas corretas ou incorretas, garantiu o anonimato dos respondentes e permaneceu disponível para os eventuais esclarecimentos das questões, reforçando a necessidade de se responder todos os itens.

\section{Tratamento dos dados}

Psicologia: Reflexão e Crítica, 2004, 17(3), pp.341-350 
Inicialmente, criou-se um banco de dados no programa SPSS, com as informações obtidas diretamente no IHS-Del-Prette (localidade, idade, sexo, momento do curso e as respostas aos 38 itens). Em seguida, calculou-se escore geral (soma das respostas aos 38 itens) e os cinco escores fatoriais de cada estudante, segundo as instruções do manual (Z. Del Prette \& A. Del Prette, 2001) bem como a identificação, separadamente por sexo, dos seus percentis correspondentes. Apesar da recomendação de que o inventário fosse respondido em sua totalidade, alguns estudantes não o fizeram e, por isso, ao se apresentar as estatísticas descritivas (valor médio e desvio-padrão), foi incluído o número de estudantes em cada caso.

Foram efetuadas análises múltiplas de variância (ANOVA) para verificar a significância dos efeitos de cada uma das variáveis independentes (localidade, sexo e idade) sobre as variáveis dependentes (escores fatoriais) e a interação entre eles. A decisão de se usar a ANOVA ao invés da MANOVA e de não se realizar uma MANOVA prévia baseou-se na análise de Hubert e Morris (1989), que questiona a adequação e a lógica do uso, bastante generalizado na pesquisa psicológica, da combinação entre MANOVA e ANOVA e explicita as condições em que um ou outro procedimento é mais adequado. Assim, apesar da correlação significativa entre as variáveis dependentes, o uso da ANOVA se justifica, neste estudo, com base em pelo menos por duas razões apontadas por aqueles autores: a) o objetivo foi comparar variáveis de resultados mais do que buscar uma combinação dessas variáveis para produzir um constructo subjacente ou um agrupamento de variáveis dependentes; b) este estudo possui uma natureza exploratória devido à escassez de pesquisas prévias sobre as relações que estão sendo investigadas e sua correspondência com constructos subjacentes.
Os efeitos significativos da ANOVA foram seguidos de análises post-hoc com contrastes entre amostras e entre subgrupos de sexo e idade (Scheffé ou o teste $t$, respectivamente, $p<0,05$ ). $\mathrm{Na}$ análise das diferenças entre os sexos, foi utilizada a versão do Teste t que leva em conta a desproporção de tamanho dos dois grupos, típica dos cursos de Psicologia. No caso da idade, cada amostra foi subdividida em dois grupos: os mais jovens (até 20 anos) e os mais velhos (acima de 20 anos), o que significou proporções que variaram de $40 \%$ a $60 \%$ de um grupo em relação ao outro.

\section{Resultados e Discussão}

Os dados descritivos gerais do repertório de habilidades sociais da amostra, subdividida por sexo, são apresentados na Tabela 3, com indicações da média, desvio-padrão e posição percentil do escore geral e dos escores fatoriais em relação aos padrões normativos disponíveis (Z. Del Prette \& A. Del Prette, 2001).

Os resultados situaram a amostra deste estudo na posição percentil mediana ou acima da mediana para o escore geral e para os escores F1, F3 e F4, com os respondentes de ambos os sexos apresentando escores abaixo da mediana no F2 e F5. Em outras palavras, em relação à amostra normativa disponível, os estudantes de Psicologia se perceberam como emitindo maior freqüência de habilidades assertivas (F1), de conversação e desenvoltura social (F3) e de auto-exposição a desconhecidos e situações novas $(\mathrm{F} 4)$ e menor freqüência das habilidades de expressão de afeto positivo (F4) e autocontrole da agressividade (F5). Pode-se concluir que, embora se avaliando como mais extrovertidos em relação às demandas de enfrentamento e

Tabela 3

Dados Descritivos ( $N=$ Número de respondentes; $M=$ Média; $D P=$ Desvio Padrão) da Amostra, por Subgrupos de Sexo, no Escore Geral e Escores Fatoriais, com Indicação da Posição Percentil em que se Situam

\begin{tabular}{llllll}
\hline \multicolumn{1}{c}{ Variáveis dependentes } & Sexo & $n$ & $M$ & $d p$ & Percentil \\
\hline \multicolumn{1}{c}{ Escore Geral } & Masculino & 97 & 96,80 & 18,03 & 51 \\
& Feminino & 445 & 94,68 & 16,61 & 58 \\
F1 - Enfrentamento e auto-afirmação com risco & Masculino & 99 & 10,61 & 3,02 & 58 \\
& Feminino & 460 & 9,21 & 2,83 & 62 \\
F2 - Auto-afirmação na expressão de afeto positivo & Masculino & 99 & 8,16 & 1,91 & 39 \\
& Feminino & 463 & 8,93 & 1,93 & 39 \\
F3 - Conversação e desenvoltura social & Masculino & 98 & 7,29 & 1,85 & 66 \\
& Feminino & 458 & 7,12 & 1,71 & 65 \\
F4 - Auto-exposição a desconhecidos e situações novas & Masculino & 98 & 3,78 & 1,12 & 68 \\
& Feminino & 464 & 3,49 & 1,21 & 53 \\
F5 - Autocontrole da agressividade em situações aversivas & Masculino & 99 & 1,21 & 0,70 & 48 \\
& Feminino & 464 & 0,93 & 0,70 & 43 \\
\hline
\end{tabular}


desenvoltura social, os estudantes de Psicologia seriam menos expressivos nos sentimentos positivos e negativos.

Em relação ao sexo, a ANOVA mostrou diferenças significativas para $\mathrm{F} 1(\mathrm{~F}=19,364 ; p=, 000) ; \mathrm{F} 2(\mathrm{~F}=12,915 ; p=, 000)$; $\mathrm{F} 4(F=4,438 ; p=, 030)$ eF5 $(F=12,967 ; p=0,000)$. A direção dessas diferenças (Teste $t$, variâncias não assumidas como iguais) foi favorável ao sexo feminino no F2 $(t=-3,614 ; p=0,000)$ e ao sexo masculino no $\mathrm{F} 1(t=4,220 ; p=0,000), \mathrm{F} 4(t=2,206 ; p=0,029)$ e F5 $(t=3,601 ; p=0,000)$. Esses resultados são semelhantes aos da amostra normativa, exceto pelo acréscimo de F4. Pode-se concluir que, embora os dois grupos sejam bastante habilidosos diante de situações que requerem "traquejo social", os do sexo masculino seriam ainda mais em relação aos do sexo feminino do que o seu grupo de referência. Por outro lado, as alunas de Psicologia não diferiram das universitárias em geral ao apresentarem maior freqüência de habilidades sociais de expressão de afeto positivo (F2) que os estudantes do sexo oposto.

A variável idade apresentou efeito significativo somente no $\mathrm{F} 4(F=6,221 ; d f=559 ; p=0,013)$, com a diferença entre os dois grupos (Testet, variâncias não assumidas como iguais) ocorrendo em direção contrária à que normalmente se esperaria com base na literatura (Caballo, 1993): os estudantes mais novos apresentaram escores mais altos que os estudantes mais velhos $(t=2,490 ; d f=564 ; p=0,019)$. Pode-se supor que, como neste estudo, a amplitude de idade foi muito pequena e que os dois grupos vivem situações e demandas razoavelmente semelhantes - outras variáveis podem estar associadas a tais efeitos.

A análise das interações entre localidade, sexo e idade mostrou-se significativa para localidade/idade $(F=1,867 ; p=0,11)$ e localidade/sexo $(F=3,791 ; p=0,10)$. A comparação entre as amostras das diferentes localidades, quanto a essas duas variáveis, é apresentada, a seguir.

Os dados descritivos dos escores fatoriais das amostras de diferentes localidades são apresentados na Tabela 4.

As diferenças entre localidades (ANOVA) foram significativas paraF1 $(F=3,721 ; p=0,011), \mathrm{F} 2(F=10,086 ; p=0,000)$ eF5 $(F=5,273$; $p=0,001)$. A análise de post-boc indicou que, no F1, os estudantes do RJ apresentaram escores mais altos que os da BA $(p=0,032) \mathrm{e}$ $\mathrm{SP}(p=0,044)$. No F2, MG apresentou escores mais altos que RJ $(p=0,000)$ e BA $(p=0,007)$. No F5, RJ apresentou escores mais baixos que MG $(p=0,012)$ e SP $(p=0,039)$. Pode-se concluir que os estudantes cariocas se percebem como mais "assertivos" que os demais e que estes seriam mais semelhantes entre si nesse aspecto. Os estudantes mineiros se avaliaram como mais

Tabela 4

Dados descritivos ( $N=$ Número de respondentes; $M=$ Média; $D P=$ Desvio Padrão) dos Escores Fatoriais de cada Amostra Estudada

\begin{tabular}{|c|c|c|c|c|c|c|c|c|c|c|c|c|}
\hline \multirow{2}{*}{$\begin{array}{c}\text { Variável } \\
\text { Dependente }\end{array}$} & \multicolumn{3}{|c|}{ MG } & \multicolumn{3}{|c|}{ BA } & \multicolumn{3}{|c|}{ SP } & \multicolumn{3}{|c|}{$\mathrm{RJ}$} \\
\hline & $\mathrm{N}$ & $\mathrm{M}$ & DP & $\mathrm{N}$ & $\mathrm{M}$ & $\mathrm{DP}$ & $\mathrm{N}$ & $\mathrm{M}$ & DP & $\mathrm{N}$ & $\mathrm{M}$ & DP \\
\hline F1 & 100 & 9,30 & 3,16 & 131 & 9,16 & 3,02 & 215 & 9,31 & 2,68 & 117 & 10,26 & 2,86 \\
\hline $\mathrm{F} 2$ & 100 & 9,51 & 1,75 & 130 & 8,54 & 1,98 & 219 & 8,93 & 1,76 & 117 & 8,17 & 2,14 \\
\hline F3 & 100 & 1,14 & ,75 & 131 & ,79 & ,79 & 219 & 1,05 & 64 & 117 & ,82 & ,60 \\
\hline F4 & 100 & 3,43 & 1,23 & 131 & 1,32 & 1,32 & 218 & 3,63 & 1,22 & 117 & 3,59 & 98 \\
\hline F5 & 100 & 1,13 & 0,75 & 131 & 0,79 & 0,79 & 219 & 1,05 & 0,63 & 117 & 82 & 0,65 \\
\hline
\end{tabular}

Tabela 5

Dados Descritivos ( $N=$ Número de respondentes; $M=$ Média) dos Escores Fatoriais de cada Amostra, Subdividida em Funcão do Sexo

\begin{tabular}{|c|c|c|c|c|c|c|c|c|c|}
\hline \multirow{2}{*}{$\begin{array}{c}\text { Variável } \\
\text { Dependente }\end{array}$} & \multirow[b]{2}{*}{ Sexo } & \multicolumn{2}{|c|}{ MG } & \multicolumn{2}{|c|}{$\mathrm{BA}$} & \multicolumn{2}{|c|}{ SP } & \multicolumn{2}{|c|}{$\mathrm{RJ}$} \\
\hline & & $\mathrm{N}$ & $\mathrm{M}$ & $\mathrm{N}$ & M & $\mathrm{N}$ & $\mathrm{M}$ & $\mathrm{N}$ & $\mathrm{M}$ \\
\hline \multirow[t]{2}{*}{$\mathrm{F} 1$} & $\mathrm{M}$ & 14 & 10,89 & 31 & 10,92 & 33 & 10,40 & 21 & 10,32 \\
\hline & $\mathrm{F}$ & 86 & 9,04 & 100 & 8,62 & 180 & 9,09 & 94 & 10,25 \\
\hline \multirow[t]{2}{*}{$\mathrm{F} 2$} & $\mathrm{M}$ & 14 & 8,40 & 31 & 7,53 & 33 & 8,78 & 21 & 7,96 \\
\hline & F & 86 & 9,69 & 99 & 8,86 & 184 & 8,97 & 94 & 8,20 \\
\hline \multirow[t]{2}{*}{ F3 } & $\mathrm{M}$ & 14 & 6,99 & 31 & 7,68 & 32 & 6,85 & 21 & 7,59 \\
\hline & F & 86 & 7,18 & 100 & 7,09 & 180 & 7,05 & 92 & 7,27 \\
\hline \multirow[t]{2}{*}{ F4 } & $\mathrm{M}$ & 14 & 3,55 & 30 & 4,12 & 33 & 3,85 & 21 & 3,34 \\
\hline & F & 86 & 3,41 & 101 & 3,26 & 183 & 3,59 & 94 & 3,65 \\
\hline \multirow[t]{2}{*}{ F5 } & $\mathrm{M}$ & 14 & 1,52 & 31 & 1,34 & 33 & 1,08 & 21 & 1,03 \\
\hline & F & 86 & 1,08 & 100 & 0,75 & 184 & 1,06 & 94 & 0,76 \\
\hline
\end{tabular}


expressivos na demonstração de sentimentos positivos em relação a amigos e familiares, aspecto em que os do RJ e BA apresentaram os mais baixos valores.

Os dados descritivos para a análise do efeito do sexo sobre os escores fatoriais, nas diferentes amostras, são apresentados na Tabela 5.

As diferenças no F1 ocorreram para a amostra de MG ( $t=$ $2,062 ; p=0,042), \mathrm{BA}(t=3,900 ; p=0,000)$ eSP $(t=2,609 ; p=0,010)$; no F4, somente para a amostra da BA $(t=3,243 ; p=0,002)$ e no F5 para BA $(t=3,812 ; p=0,000)$ e MG $(t=2,063 ; p=0,042)$. As diferenças no $\mathrm{F} 2$, favoráveis ao sexo feminino, foram verificadas para a amostra de MG $(t=2,630 ; p=0,010)$ e da BA $(t=-3,394$; $p=0,001)$.

Com base nesses dados, podemos concluir que a influência do sexo parece ser maior entre os estudantes de Psicologia da Bahia e, em segundo lugar, entre os estudantes de Minas, onde ocorreu para maior número de fatores. As diferenças verificadas coincidem com as da amostra geral e da normativa em que se apóia o IHS-Del-Prette (Z. Del Prette \& A. Del Prette, 2001), ou seja, superioridade masculina em F1 e F5 e feminina no F2, exceto que, entre os da Bahia, os homens apresentaram escores fatoriais significativamente maiores também no F4. É interessante observar que houve apenas uma diferença significativa de sexo para a amostra de São Paulo, referente a F1 e nenhuma entre os estudantes do Rio de Janeiro.

Os dados descritivos para a análise do efeito da idade sobre os escores fatoriais, nas diferentes localidades, são apresentados na Tabela 6.

Como se vê na Tabela 6 , os dados descritivos das duas faixas de idade são, nominalmente, bastante semelhantes nas quatro amostras. A análise estatística das diferenças de idade foi favorável aos mais novos no F4 somente entre os estudantes de SP. Adicionalmente, foram encontradas duas diferenças no F5, porém favoráveis aos mais velhos: na amostra da BA $(t=-2,158 ; p=0,032)$ e do RJ $(p=-2,36 ; p=0,022)$.

\section{Conclusão}

Os resultados encontrados indicaram mais semelhanças que diferenças entre as amostras, em termos do padrão de desempenho social aferido pelo IHS-Del-Prette (freqüência de emissão de reações socialmente competentes). Cabe, no entanto, destacar que, neste estudo, a afirmação de semelhanças ou diferenças não inclui os aspectos formais do desempenho das habilidades avaliadas (componentes não verbal e paralingüístico de cada uma das habilidades sob análise) nem sua proficiência (enquanto indicador competência social), mas somente o relato de freqüência. Embora a freqüência seja uma das dimensões utilizadas para a avaliação do repertório das habilidades sociais, é sempre importante reconhecer os limites de cada um dos indicadores produzidos pelos diferentes instrumentos no contexto da multidimensionalidade do desempenho social (A. Del Prette \& Z. Del Prette, 1999; Z. Del Prette \& A. Del Prette, 2001, 2002).

Chama à atenção o padrão geral dos estudantes de psicologia investigados, quanto à menor expressividade de sentimentos positivos (F2) e negativos (F5) em relação à amostra normativa de universitários, o que se mostrou particularmente crítico para as amostras do RJ (F2 e F5) e BA (F5). Pode-se supor, aqui, pelo menos duas explicações: a) um ajustamento do estudante ao estereótipo do psicólogo como um profissional mais contido e controlado, que poderia ser reforçado ao longo da graduação ou, mesmo, constituir um fator pré-seletivo dos que buscam essa área de formação; b) déficits de habilidades sociais nessa área, que o curso não estaria suprindo (Ex.: ver Z. Del Prette, A. Del Prette \& Branco, 1992a) embora focalizando outras características do desempenho social. Essas hipóteses poderiam

Tabela 6

Dados Descritivos ( $N=$ Número de respondentes; $M=$ Média) dos Escores Fatoriais de cada Amostra, Subdividida em Função da Faixa Etária

\begin{tabular}{|c|c|c|c|c|c|c|c|c|c|}
\hline \multirow{2}{*}{$\begin{array}{c}\text { Variável } \\
\text { Dependente }\end{array}$} & \multirow[b]{2}{*}{ Grupo } & \multicolumn{2}{|c|}{ MG } & \multicolumn{2}{|c|}{$\mathrm{BA}$} & \multicolumn{2}{|c|}{ SP } & \multicolumn{2}{|c|}{$\mathrm{RJ}$} \\
\hline & & $\mathrm{N}$ & M & $\mathrm{N}$ & M & $\mathrm{N}$ & M & $\mathrm{N}$ & M \\
\hline \multirow[t]{2}{*}{ Escore Geral } & $\leq 20$ & 38 & 99,34 & 58 & 92,05 & 120 & 94,95 & 40 & 97,45 \\
\hline & $>20$ & 62 & 94,35 & 67 & 96,09 & 84 & 91,62 & 41 & 97,94 \\
\hline \multirow[t]{2}{*}{$\mathrm{F} 1$} & $\leq 20$ & 38 & 9,59 & 58 & 8,69 & 129 & 9,55 & 41 & 10,56 \\
\hline & $>20$ & 62 & 9,13 & 73 & 9,55 & 86 & 8,96 & 76 & 10,10 \\
\hline \multirow[t]{2}{*}{$\mathrm{F} 2$} & $\leq 20$ & 38 & 9,82 & 59 & 8,42 & 131 & 8,99 & 41 & 7,81 \\
\hline & $>20$ & 62 & 9,33 & 71 & 8,65 & 88 & 8,84 & 76 & 8,37 \\
\hline \multirow[t]{2}{*}{ F3 } & $\leq 20$ & 38 & 7,39 & 59 & 7,16 & 127 & 7,08 & 41 & 7,08 \\
\hline & $>20$ & 62 & 7,00 & 72 & 7,28 & 87 & 6,91 & 76 & 7,48 \\
\hline \multirow[t]{2}{*}{ F4 } & $\leq 20$ & 38 & 3,50 & 59 & 3,54 & 131 & 3,77 & 41 & 3,72 \\
\hline & $>20$ & 62 & 3,39 & 72 & 3,38 & 87 & 3,43 & 76 & 3,52 \\
\hline \multirow[t]{2}{*}{ F5 } & $\leq 20$ & 38 & 1,14 & 59 & 0,73 & 131 & 1,08 & 41 & 0,63 \\
\hline & $>20$ & 62 & 1,14 & 72 & 1,02 & 88 & 1,02 & 76 & 0,92 \\
\hline
\end{tabular}


ser investigadas em estudos futuros, comparando-se o padrão apresentado pelos iniciantes e concluintes.

Essas diferenças poderiam também refletir valores e normas próprios dessas duas localidades que têm, em comum, o fato de serem cidades de grande porte, ao contrário das duas outras. Aqui, é interessante ressaltar que a amostra do RJ apresentou os escores mais altos nas habilidades assertivas de enfrentamento (F1), configurando portanto, um certo desequilibrio entre a competência diante de demandas negativas e positivas. Pode-se levantar a hipótese de que, em grandes metrópoles em geral, e no Rio de Janeiro em particular, o estresse cotidiano, que gera maior preocupação com a integridade e segurança, pode restringir a expressividade de sentimentos positivos. Esta é uma questão a ser investigada comparando-se amostras de cidades grandes e pequenas.

O padrão de semelhanças identificado neste trabalho ocorreu tanto para o escore geral como para os escores fatoriais, sugerindo que a base cultural comum a essas localidades é mais determinante do que as eventuais diferenças entre subculturas, pelo menos em termos do auto-relato da freqüência de habilidades sociais. Essa base cultural comum certamente está relacionada a vários fatores, entre eles: a) a semelhança da língua, que estabelece um conjunto de regras para a comunicação, aqui se incluindo as formas de comunicação assertiva (Hargie, Saunders \& Dickson, 1994); b) a alta migração e mobilidade populacional, gerando forte mesclagem de costumes e formas de relacionamento; c) os meios de comunicação que realçam e disseminam certos padrões de interação social. As telenovelas, por exemplo, oferecem modelos de desempenhos para diferentes situações, bem como variantes para pessoas de diferentes características demográficas. No caso das amostras situadas na faixa etária deste estudo, para as quais são bastante críticas as demandas afetivas, de trabalho e de constituição de vida independente, tais modelos podem ser especialmente efetivos.

Apesar do padrão geral de semelhanças, é interessante observar que há mais semelhança entre São Paulo e Minas e entre Rio de Janeiro e Bahia, do que entre esses dois subgrupos. No escore geral, enquanto os estudantes do Rio de Janeiro apresentaram valores mais altos no F1, os de Minas se destacaram no F2, sugerindo uma possível influência das características urbanas de cidades maiores, enquanto capitais, em relação às menores e de interior.

Um outro aspecto importante na análise das diferenças entre localidades foi o sexo, o que é coerente com estudos que situam essa variável como um importante moderador (no sentido proposto por Baron \& Kenny, 1986) de diferenças ou semelhanças no desempenho social entre culturas (Fray \& Hector, 1987). As diferenças encontradas revelam o peso do conceito de assertividade em F1 e F5 e situam os homens como mais assertivos que as mulheres, conforme verificado em outros países (Burgoon, Dillard \& Doran, 1984; Chandler \& cols., 1978;
Nicotera \& Rancer, 1994; Margalit \& Eysenck, 1990; Sarason, Sarason, Hacker \& Basham, 1985, entre outros).

No entanto, considerando-se o efeito do sexo nas diferentes amostras, verificou-se que São Paulo e Rio de Janeiro apresentaram um padrão menos diferenciado entre homens e mulheres do que as duas amostras restantes. É dificil explicar esse resultado, porém pode-se indagar se a educação dos jovens de RJ e SP viria incorporando as noções de igualdade de gênero mais fortemente do que os jovens das outras localidades estudadas.

Dada a influência do sexo, pode-se dizer que, quando essa variável não foi controlada (caso de localidade e idade) os resultados acabaram refletindo mais fortemente o padrão feminino, típico dos estudantes de Psicologia. Embora a escolha do curso fosse motivada pela preocupação de controle dessa variável, pode-se esperar resultados diferentes em estudos futuros com estudantes de cursos onde essa composição seja mais equilibrada.

$\mathrm{Na}$ análise da influência da idade, verificou-se que praticamente não ocorreram diferenças. Pode-se levantar aqui duas hipóteses: a) a faixa etária contemplada neste estudo não foi suficientemente ampla para diferenciar os dois subgrupos; b) tais diferenças passam a ser menores a partir da adolescência. Esses aspectos sugerem questões para pesquisas adicionais tais como a identificação dos períodos do ciclo vital em que essas diferenças são mais salientes e até mesmo a possibilidade de uma assíntota em determinada faixa etária.

Em resumo, os dados mostraram um padrão amplo de semelhanças, com algumas diferenças somente em aspectos pontuais, fornecendo indicadores adicionais de validade para $\mathrm{o}$ IHS-Del-Prette, pelo menos junto a estudantes de Psicologia, o que não dispensa a necessidade de novos estudos deste instrumento, seja com a finalidade de confirmar sua estrutura dimensional em amostras oriundas de outras localidades, seja com o objetivo prático de ampliar os parâmetros normativos para sua utilização na avaliação clínica.

O exame mais detalhado do significado das diferenças encontradas neste estudo certamente envolveria, ainda, a análise adicional das outras dimensões do conceito de competência social, o que poderia ser feito com o uso de outros instrumentos (como a entrevista semi-estruturada) ou mesmo com questões sobre os itens do IHS-Del-Prette, que permitissem o acesso indicadores adicionais. Poder-se-ia, por exemplo, em uma segunda etapa deste estudo, examinar a percepção dos respondentes sobre o grau de ansiedade que experimentam diante das situações descritas nos itens. Adicionalmente, verificar suas atribuições quanto à adequação de reações socialmente competentes e não competentes ou, ainda, suas avaliações de proficiência para lidar com tais situações. Considerando-se a diversidade dessas dimensões e a ocorrência de correlação entre os fatores (na dimensão freqüência), seria interessante investigar, em estudos futuros, por meio de análises multivariadas, a composição dessas dimensões em possíveis constructos subjacentes, como forma 
de aprofundar a análise das diferenças entre essas amostras e subgrupos.

A natureza situacional-cultural das habilidades sociais tem sido amplamente reconhecida na literatura (Argyle, 1984; 1994; Bryant, \& Trower, 1974; Furnham, 1979; Trower \& cols., 1978, entre outros), mas pode-se argumentar que é ainda insuficientemente sustentada por dados empíricos sobre padrões característicos do desempenho social de diferentes grupos culturais. Este estudo, ainda que essencialmente exploratório e preliminar em nosso país, constitui uma tentativa de contribuição para a ampliação de tais dados. Entende-se que um maior investimento nessa temática remete não somente a questões metodológicas ligadas ao aperfeiçoamento de instrumentos de avaliação, mas também a questões relativas à própria estrutura dos conceitos de habilidades sociais e de competência social.

\section{Referências}

Abarca, N. \& Hidalgo, C. G. (1989). Evaluación psicométrica de habilidades sociais en jovens universitarios Chilenos. Revista Análisis del Comportamiento, 4, 51-62.

Argyle, M. (1984). Some new developments in social skills training. Bulletin of British Psychological Society, 37, 405-410.

Argyle, M. (1988). Bodily communication (2 ${ }^{a}$ ed.). Methuen: London

Argyle, M. (1994). Psicologia del comportamiento interpersonal. Madrid: Alianza Universidad. (Original publicado em 1967)

Ayres, L. S. M. (1994). Uma escala brasileira para a medida da assertividade. Dissertação de Mestrado não-publicada, Universidade Gama Filho. Rio de Janeiro, RJ.

Bandeira, M. (1999). Competência social de psicóticos: Parâmetros de treinamento para programas de reabilitação psicossocial (Parte II). Jornal Brasileiro de Psiquiatria, 48, 191-195.

Bandeira, M. \& Tremblay, L. (1998). Competência social de psicóticos: Um estudo de validação social. Jornal Brasileiro de Psiquiatria, 47, 185-192.

Bandeira, M., Costa, M. N., Del Prette, Z. A. P., Del Prette, A. \& Gerk-Carneiro, E. (2000). Qualidades psicométricas do Inventário de Habilidades Sociais (IHS): Estudo sobre a estabilidade temporal e a validade concomitante. Estudos de Psicologia, 5, 401-419.

Baron, R. M. \& Kenny, D. A. (1986). The moderator-mediator variable distinction in social psychological research: Conceptual, strategic and statistical considerations. Journal of Personality and Social Psychology, 51, 1173-182.

Bresnahan, M. J., Shearman, S. M. \& Lee, S. Y. (2002). Personal and cultural differences in responding to criticism in three countries. Asian Journal of Social Psychology, 5, 93-105.

Bryant, B. \& Trower, P. (1974). Social difficulty in a student sample. British Journal of Educational Psychology, 44, 13-21.

Bueno,J. M. H., Oliveira, S. M. S. S. \& Oliveira, J. C. S.(2001). Um estudo correlacional entre habilidades sociais e traços de personalidade com universitários. PsicoUSF, 6, 31-38.

Burgoon, M., Dillard,J. P. \& Doran, N. E. (1984). Friendly or unfriendly persuasion: The effects of violation of expectations by males and females. Human communication Research, 10, 283-294.

Caballo, V. E. (1993). Manual de evaluación y entrenamiento en habilidades sociales. Madrid: Siglo Veintiuno.

Caballo, V. E. (1995). Una aportación española a los aspectos moleculares, a la evaluación y al entrenamiento de las habilidades sociales. Revista Mexicana de Psicologia, 12, 121-131.

Carmona, A. E., \& Lorr, M. (1992). Dimensions of assertiveness: A cross-cultural comparison of Chilean and U. S. subjects. Personality \& Individual Differences, $13,45-48$
Chandler, T., Cook, B. \& Dugovic, D. (1978). Sex differences in self-reported assertiveness. Psychogical Reports, 43, 395-402.

Del Prette, A. (1978). O treino assertivo na formação do psicólogo. Arquivos Brasileiros de Psicologia Aplicada, 30, 53-55.

Del Prette, A. \& Del Prette, Z. A. P. (2001). Psicologia das relações interpessoais: Vivências para o trabalho em grupo. Petrópolis: Vozes.

Del Prette, A., Del Prette, Z. A. P. \& Barreto, M. C. M. (1999). Habilidades sociales en la formación del psicólogo: Análisis de un programa de intervención. Psicología Conductual, 7, 27-47.

Del Prette, A., Del Prette, Z. A. P. \& Branco, U. V. C. (1992). Competência social na formação do psicólogo. Paidéia: Cadernos de Educação, 2, 40-50.

Del Prette, Z. A. P. \& Del Prette, A. (1983). Análise de repertório assertivo em estudantes de Psicologia. Revista de Psicologia, 1, 15-24.

Del Prette, Z. A. P. \& Del Prette, A. (1999). Psicologia das habilidades sociais: Terapia e educação. Petrópolis: Vozes.

Del Prette, Z. A. P. \& Del Prette, A. (2001). Inventário de Habilidades Sociais (IHS-DelPrette): Manual de aplicação, apuração e interpretação. São Paulo: Casa do Psicólogo.

Del Prette, Z. A. P. \& Del Prette, A. (2002). Avaliação de habilidades sociais de crianças com um inventário multimídia: Indicadores sociométricos associados à freqüência versus dificuldade. Psicologia em Estudo, 7(1), 61-73.

Del Prette, Z. A. P., Del Prette, A. \& Branco, U. V. C. (1992). Competência social: Um levantamento de situações críticas de interação para estudantes de Psicologia. Em Associação Brasileira de Psicologia Escolar e Educacional (Org.), Psicólogo Escolar: Identidade e Perspectivas: Anais do I Congresso Nacional de Psicologia Escolar $e$ Educacional. (pp. 384-387). Campinas: ABRAPEE.

Del Prette, Z. A. P., Del Prette, A. \& Correia, M. F. B. (1992). Competência social: Um estudo comparativo entre alunos de Psicologia, Serviço Social \& Engenharia Mecânica. Psicólogo Escolar: Identidade e Perspectivas, 382-384.

Falcone, E. O. (1998). Avaliação de um programa de treinamento de empatia com universitários. Tese de Doutorado não-publicada, Instituto de Psicologia, Universidade de São Paulo. São Paulo, SP.

Fray, J. S., \& Hector, M. A. (1987). The assertive-aggressive distinction and the cross-cultural perspective. International Journal for the Advancement of Counselling, 10, 103-110.

Furnham, A. (1979). Assertiveness in three cultures: Multidimensionality and cultural differences. Journal of Clinical Psychology, 35, 522-527.

Furnham, A. (1993). Communication in foreign lands: The cause, consequences and cures of culture schock. Language, Culture and Curriculum, 6, 91-109.

Galassi, J. P., Delo, J. S., Galassi, M. D. \& Bastien, S. (1974). The college selfexpression scale: A measure of assertiveness. Behavior Therapy, 5, 165-171.

Gerk-Carneiro, E., Alves, L. H. J., Ziviani, C. R., Barros, A. M. M., Takahashi, F. D. M. \& Araújo, L. B. (2001a). Estudo comparativo de duas escalas de habilidades sociais [Resumos]. Em Sociedade Brasileira de Psicologia (Org.), Resumos da XXXI Reunião Anual da sociedade Brasileira de Psicologia (p. 42). Rio de Janeiro: SBP.

Gerk-Carneiro, E., Alves, L. H. J., Ziviani, C. R., Barros, A. M. M., Takahashi, F. D. M. \& Araújo, L. B. (2001b). Sondagem das Habilidades Sociais em Estudantes Universitários Brasileiros. Em Actas do VI Congresso Galaico-Português de Psicopedagogia (Vol. II, pp. 949-962). Braga: Centro de estudos de Educação e Psicologia.

Hall, E. T. (1977). A dimensão oculta (2 $2^{\mathrm{a}}$ ed.). Rio de Janeiro: Francisco Alves.

Hargie, O., Saunders, C. \& Dickson, D. (1994). Social skills in interpersonal communication ( $3^{\mathrm{a}}$ ed.). London: New York: Routledge. (Original publicado em 1981)

Hidalgo, C. G. \& Abarca, N. (1990). Desarollo de habilidades sociales en estudiantes universitarios. Revista Latinoamericana de Psicologia, 22, 2165-282.

Hubert, C. \& Morris, J. (1989). Multivariate versus multiple univariate analysis. Psychological Bulletin, 105, 302-308.

LaFrèniere, P., Masataka, N., Butovskaya, M., Chen, Q., Dessen, M. A., Atwanger, K., Schreiner, S., Montirosso, R. \& Frigerio, A. (2002). Cross-cultural analysis of social competence and behavior problems. Early education \& Development, 13,201-219.

Lonner, W. J., Dinnel, D. L., Hayes, S. A. \& Sattler, D. N. (Orgs.) (2002). Online readings in Psychology and Culture [bttp:/ / mnn.wnu.edu/ culture]. Center for Cross- 
Zilda A P. Del Prette, Almir Del Prette, Marina Bandeira, Maria Refugio Rios-Saldaña, Ana Lucia Alcântara Oliveira Ulian, Eliane, Gerk-Carneiro, Eliane Mary de O. Falcone,

350 Maria Cecília Mendes Barreto \& Miriam Bratfisch Villa

Cultural Research, Western Washington University, Bellingham, Washington, USA.

Margalit, M. \& Eysenck, S. (1990). Prediction of coherence in adolescence: Gender differences in social skills, personality, and family climate. Journal of Research in Personality, 24, 510-521.

McFall, R. M. (1982). A review and reformulation of the concept of social skills. Behavioral Assessment, 4, 1-33.

Nicotera, A. M. \& Rancer, A. S. (1994). The influence of Sex on self-perceptions and social stereotyping of aggressive communication predispositions. Western Journal of Communication, 58, 283-301.

Nikura, R. (1999). The psychological process underlying Japanese assertive behavior: Comparison of Japanese with Americans, Malaysians and Filipinos. International Journal of Intercultural Relations, 23, 47-76.
Rathus, S. A. (1973). A 30-itemschedule for assessing assertive behavior. Behavior Therapy, 4, 398-406.

Sarason, B. R., Sarason, I. G., Hacker, T. A. \& Basham, R. B. (1985). Concomitants of social support: Social skills, physical attractiveness, and gender. Journal of Personality and Social Psychology, 49, 469-480.

Trower, P., Bryant, B. \& Argyle, M. (1978). Social skills and mental health. London: Methuen.

Wood, P. S. \& Mallinckrodt, B. (1990). Culturally sensitive assertiveness training for ethnic minority clients. Professional Psychology: Research and Practice, 21, 5-11.

Yashima, T. \& Tanaka, T. (2001). Roles of social support and social skills in the intercultural adjustment of Japanese adolescents sojourners in USA. Psycbological Reports, 3(2), 1201-1210.

Sobre os autores

Zilda A P. Del Prette é Psicóloga, Pós-doutora pela University of Califórnia, Berkeley. É Professora da Universidade Federal de São Carlos.

Almir Del Prette é Psicólogo, Doutor em Psicologia pela Universidade de São Paulo. É Professor da Universidade Federal de São Carlos.

Marina Bandeira é Pós-doutora pelo Centre de Recherche Fernand Seguin - Université de Montreal, Canadá. É Professora da Universidade Federal de São João Del Rei.

Maria Refugio Rios-Saldaña é Professora da Universidad Nacional Autônoma do México.

Ana Lúcia Alcântara Oliveira Ulian é Psicóloga e Doutoranda em Psicologia Clínica pela Universidade de São Paulo. É Professora da Universidade Federal da Bahia.

Eliane Gerk-Carneiro é Psicóloga, Doutora em Psicologia pela Fundação Getúlio Vargas. É Professora da Universidade Estácio de Sá.

Eliane Mary de Oliveira Falcone é Doutora em Psicologia Clínica pela Universidade de São Paulo. É Professora da Universidade Estadual do Rio de Janeiro.

Maria Cecília Mendes Barreto é Estatística e Pós-doutora pela The University Of Nottingham, Inglaterra. É Professora da Universidade Federal de São Carlos.

Miriam Bratfisch Villa é Psicóloga e Doutoranda em Psicologia pela Universidade de São Paulo, Ribeirão Preto. 